\title{
Architecture of a Multiple Channel Blood Pressure Signal Transmitting Based on Bluetooth Technology
}

\author{
Ruirui $\mathrm{He}^{1, \mathrm{a}}$, Lina Wang ${ }^{2}$, Jianqiang $\mathrm{Du}^{2}$, Xiaoming $\mathrm{Wu}^{2, \mathrm{~b}}$ \\ ${ }^{1}$ Department of Industrial Automation, School of Electrical Engineering, Xi'an Jiaotong University, \\ Xi'an, 710049, China \\ ${ }^{2}$ The Key Laboratory of Biomedical Information Engineering of Ministry of Education, School of Life \\ Science and Technology, Xi'an Jiaotong University,Xi'an, 710049, China \\ aemail: 13363991221@163.com, bemail:wxm@mail.xjtu.edu.cn
}

Keywords: Blood Pressure; Bluetooth; Real-time Measurement; CC2540

\begin{abstract}
Recent technological advancements have led to the production of microcontroller with wireless communication functions, which enable the integration of physiological data collection, data processing, and data transmitting. Blood pressure is an important physiological indicator which should be measured continuously in some case. In this article, we bring forward an architecture including pressure measure based on a fiber optic sensor, data transmitting using Bluetooth technology, and data collection using a Bluetooth-Ethernet gateway running protocol process software. Particularly, a control chip CC2540 was used to run the Bluetooth protocol and to make the data transmitting, a module based on W5100 chip was used to realize TCP/IP protocol. The blood pressure data was finally send to a database server through the Ethernet interface, to make further analysis and data mining.
\end{abstract}

\section{Introduction}

One of the most widespread and important applications in wireless sensor networks (WSNs) is the continuous data collection, such as monitoring the variety of ambient temperature and humidity[1]. Blood pressure real-time detection has important usage in medical research also. Traditional blood pressure measurement can't get continuous data. By using fiber optical blood pressure sensors, the signal can be recorded, and then transmitted out through the wireless network, so as to achieve a real-time, fast and multiplex transmission effect. Recent technological advancements have led to the production of miniaturized sensors, by using a wide range of radio standards [2]. Research also shows that telehealth is usable in clinical data collection such as blood glucose monitor[3], or EEG Signals collection[4]. Bluetooth 4.0 technology provides communication within 10 meter distance by using low energy consumption, and it is the ideal choice in this study to construction a blood pressure detection network.

In this paper, we illustrate an overall scheme including the design of pressure sensors module, the wireless communication consideration, and data storage method. The wireless pressure sensor network is based on Bluetooth 4.0 protocol, an Ethernet module is used to serve as Bluetooth gateway to transmit data through TCP/IP protocol, by using a W5100 micro controller. The gateway also does the data collection by large-scale accessions to real-time measurement of blood pressure data.

\section{Principle of Blood Pressure Measurement}

The blood pressure sensor (probe) is based on a micro Fabry-Perot cavity at the end of an optical fiber. A work module generate infrared laser, transmit the laser through optical fiber and reach the sensor, which is also a portion of the optical fiber. Pressure around the probe changes the reflected laser intensity. Through the optical fiber, the reflected light reached a photoelectric transducering element, where a voltage signals produced. After calibration, the voltage signal will embody the change of blood pressure dynamically. The pressure sensor module contain several functional parts, including laser emission, photoelectric conversion, signal amplification, modulus conversion, data 
processing, etc.. This part generates digital blood pressure signals, which was achieved by using micro controller such as a MSP430, together with analog circuits.

Bluetooth Protocol. Bluetooth 4.0 includes Bluetooth Low Energy (BLE) protocol, and Classic Bluetooth protocol. BLE is a short-range wireless communication technology aiming at low-cost and low-power communication [5]. It is intended to provide reduced power consumption while maintaining a similar communication range. A single button battery can work the BLE device for a year. A group of connected BLE devices can make up a piconet, which can include up to one master device and 7 slave devices. In the piconet, each device is assigned a specific time period to transmit, so the data will not collide each other when data transferring. Data transfer rates can vary between about 200 and 2100 kilobits per second.

To implement Bluetooth protocol, heavy data processing is needed to make decoding, encoding and signal transmitting. Usually, specialized module is used to make the implement. In this study, the sensor get the blood pressure, the module make the measurement and data convert, and then send to the Bluetooth module. This module transmits the data through wireless connection, and send to the Bluetooth gateway. At the same time, up to 7 devices can connect to the gateway and transmitting real time data.

Bluetooth Chip. The TI CC2540 is a low-power, system-on-chip (SoC) for BLE applications. It enables robust BLE master or slave nodes to be built. It combines an RF transceiver with an 8051 MCU, in-system programmable flash memory, 8-KB RAM, and other supporting features and peripherals. In this study, the sensor module is composed of a CC2540 SoC, and peripheral circuits, which made data acquisition and implementing the wireless communication.

W5100. The W5100 is a single-chip Internet-enabled 10/100 Ethernet controller designed for embedded applications. It has been designed to facilitate easy implementation of Internet connectivity. The W5100 includes fully hardwired, market-proven TCP/IP stack and integrated Ethernet MAC \& PHY. It does not need of consideration for handling Ethernet controller, but only simple socket programming is required. Three different interfaces like memory access way, called direct, indirect bus and SPI, are supported on the MCU side. The W5100 is suited for many embedded applications, such as Home Network Devices (HND), Medical Monitoring Equipment (MME), Embedded Servers (ES) and others. Based on W5100, it is easy to make data transmitting and communication.

\section{The Architecture of the System}

The whole system consists of three parts, which are pressure detection part, Bluetooth gateway part, and data storage server part. Within pressure detection part, there are laser emission module, photo detection module, micro controller, and Bluetooth module. This part measures the pressure of biological object, converts the pressure into numerical data, and transmits the data through Bluetooth protocol. Each biological object would equip with one device containing the pressure detection part. This device is moveable, so it can attach to the body of the animal under investigating. The overall diagram is illustrated in figure 1.

From the diagram we can see, Bluetooth gateway collects the data sent from sensor modules, each gateway can connect to 7 sensors from 7 channels simultaneously. It also transmits data to database server through Ethernet. the pressure sensing part has a weak data processing capacity, since it just make data transmitting, while the Bluetooth gateway has a slightly strong processing capacity, due to more data processing needed, and the need of protocol conversion. The database server has the most powerful data processing ability. 

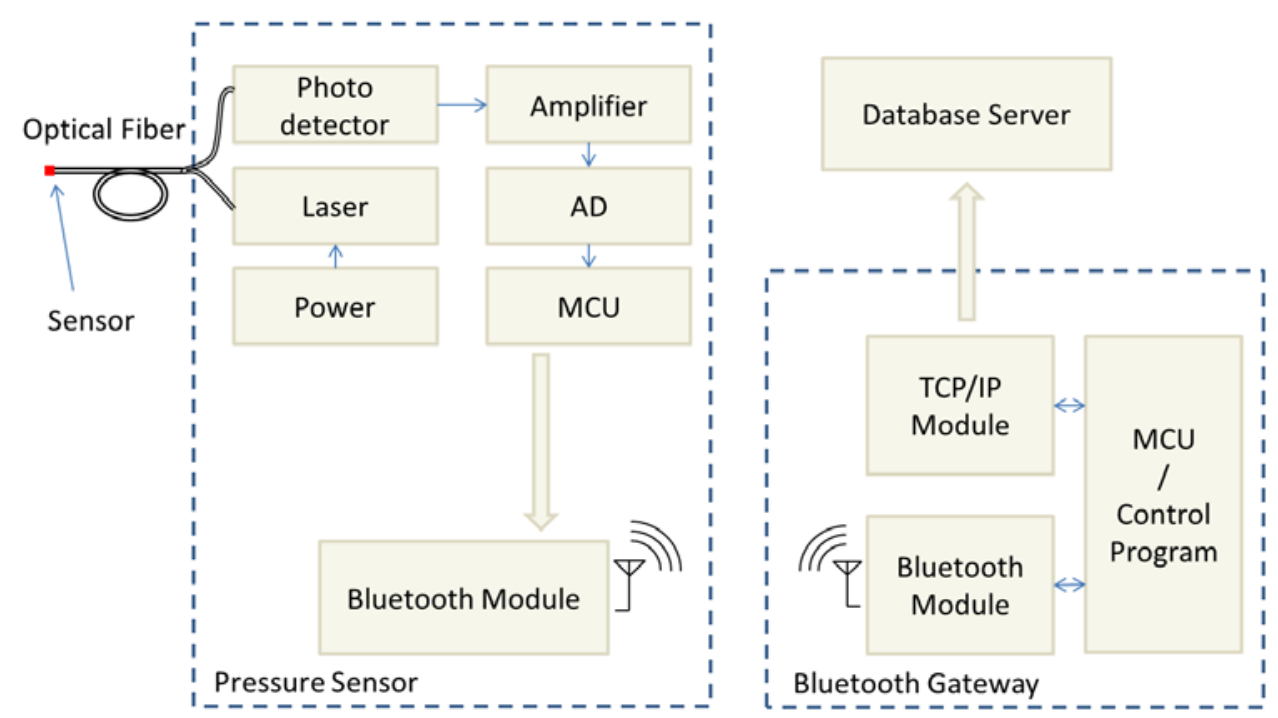

Fig. 1: Overall system framework

\section{The Function of Modules}

Pressure Sensors. Optical fiber based sensor can convert pressure into light intense change, and is used for blood pressure measure in this research. In pressure sensor part, electric circuit is adopted to convert light into voltage signal and make amplify; an MCU is used to mapping the voltage value into pressure, to realize the data acquisition. Through communication, the MCU transmit the data to Bluetooth module, which in turn send those data to Bluetooth gateway. This part is also a Bluetooth node equipment.

Bluetooth Gateway. Bluetooth gateway will establish connections through Bluetooth protocol, and made data transmission through TCP/IP protocol. The gateway contains a Bluetooth wireless transceiver which processes and reformats pressure data as Internet/Ethernet accessible formats. It has the advantage that high performance computer based on TCP/IP protocol can make use of the sensor data for data visualization and data management. In this part, CC2540, W5100, together with a MCU was included. The communication with in the part was based on SPI serial bus.

\section{System Software Design}

Considerations of Bluetooth wireless network. The Bluetooth gateway is the main part in this design. It can connect to 7 Bluetooth nodes simultaneously, and thus a network constructed. When one node in the network finishes transmitting data, the controller disconnected the connection, and a new node can add to the network, thus the number of nodes in the network can be increased. According to currently protocol, 7 nodes can be added to the network.

Ethernet Data Transmitting. TCP is a connection based protocol, the IP address, the port number should be allocated, and a connection path should establish before the data transmitting process begin. In this research, w5100 chip is served as a TCP client, which requests for a connection from a known server, and then made data upload. In this case, the TCP gateway, subnet mask, physical address, buffer size and other parameter should set before hand. A micro controller connects to w5100 through SPI data bus, which contains two data lines for data receiving and sending, and one clock line indicate data duration. The overall diagram of system software is show in figure 2 . 


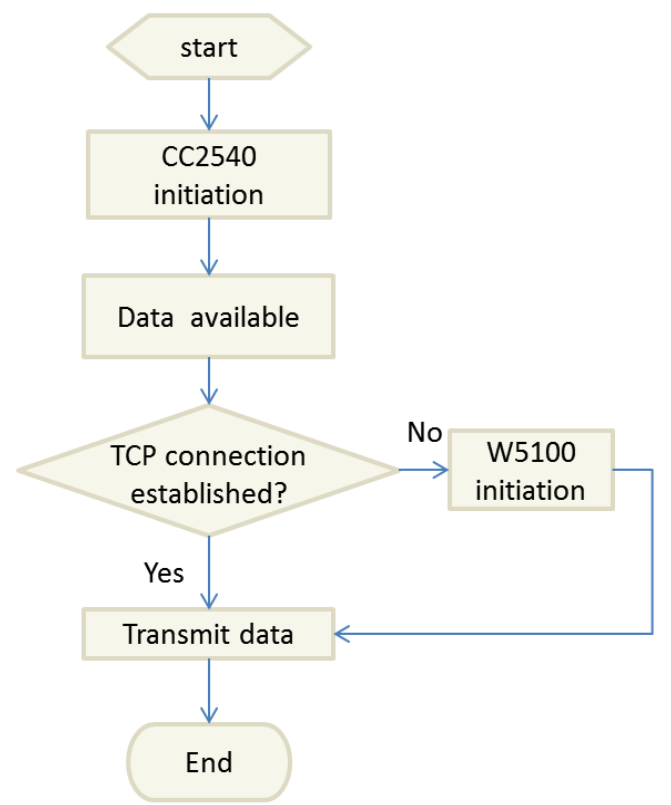

Figure 2: Flow chart for data receiving and transmitting

A microcontroller runs program and controls the start of Bluetooth protocol, the TCP/IP protocol, and manage the data transmitting.

\section{Summary}

Bluetooth 4.0 technology has the advantage of low power consumption, low cost, AES-128 security encryption, and many other features, through the frequency hopping technology, it has the anti-jamming feature to establish reliable wireless network. This paper designed a multi-channel blood pressure measurement system based on Bluetooth technology. Each blood pressure measurement node is related to one biological individual to test blood pressure. At the same time, it makes connection to Bluetooth gateway. Each gateway can connect to 7 Bluetooth devices simultaneously. Through Ethernet, The blood pressure information was sent to the databases server. This scheme is mainly used for animal blood pressure monitoring.

\section{Acknowledgements}

This work was financially supported by the National Natural Science Foundation of China (81102085), the Fundamental Research Funds for the Central Universities (2012jdhz47, xjj2013117), and the Shaanxi Science and Technology Plan Projects (2011k12-4-6).

\section{References}

[1] Wu, M., L. Tan, and N. Xiong, A structure fidelity approach for big data collection in wireless sensor networks. Sensors (Basel), 2015. 15(1): p. 248-73.

[2] Kotsev, A., et al., Architecture of a service-enabled sensing platform for the environment. Sensors (Basel), 2015. 15(2): p. 4470-95.

[3] Welch, G., A. Balder, and S. Zagarins, Telehealth program for type 2 diabetes: usability, satisfaction, and clinical usefulness in an urban community health center. Telemed J E Health, 2015. 21(5): p. 395-403.

[4] Pastena, L., et al., Bluetooth Communication Interface for EEG Signal Recording in Hyperbaric Chambers. IEEE Trans Neural Syst Rehabil Eng, 2015.

[5] Cho, K., et al., Analysis of latency performance of bluetooth low energy (BLE) networks. Sensors (Basel), 2015. 15(1): p. 59-78. 